\title{
Relationship between blood-brain permeability and antibodies against aquaporins in neuromyelitis optica spectrum disorders and multiple sclerosis patients
}

\author{
Michalina Jasiak-Zatońska ${ }^{1}$, Sławomir Michalak ${ }^{1}$, Krystyna Osztynowicz $^{1}$, \\ Wojciech Kozubski², Alicja Kalinowska-Łyszczarz ${ }^{1}$ \\ ${ }^{1}$ Department of Neurochemistry and Neuropathology, Poznan University of Medical Sciences, Poznan, Poland \\ ${ }^{2}$ Department of Neurology, Poznan University of Medical Sciences, Poznan, Poland
}

\begin{abstract}
Aim of the study. To evaluate serum anti-aquaporin antibodies profile, to measure serum levels of cell-cell adhesion molecules as potential markers of blood-brain barrier (BBB) permeability, and to assess their relationship in neuromyelitis optica spectrum disorders (NMOsd) and multiple sclerosis (MS).

Clinical rationale for the study. Serum levels of cell-cell adhesion molecules could provide information about BBB disruption in demyelinating diseases of the central nervous system. Improved knowledge about differences in their profile in NMOsd and MS patients, as well as about their relationship with antibody serostatus, would facilitate early and accurate diagnosis.

Material and methods. Sera from 20 NMOsd and 59 MS patients were collected and assessed for anti-aquaporin 4 antibodies (AQP4-IgG) and antibodies against myelin oligodendrocyte glycoprotein (MOG-Ab) using an indirect immunofluorescence test (IIFT). Anti-aquaporin 1 antibodies (AQP1-Ab), vascular endothelial growth factor (VEGF), and vascular endothelial cadherin (VE-Cadherin) levels were assessed using commercial enzyme-linked immunosorbent assay (ELISA) kits. For occludin (OCLN) and claudin-5 (CLDN5) serum levels, we employed home-made ELISAs elaborated in the Department of Neurochemistry and Neuropathology, Poznan University of Medical Sciences, Poland.

Results. AQP4-IgG appeared only in 6/20 NMOsd patients who were all originally AQP4-lgG seropositive. All MS and NMOsd patients were seronegative for MOG-Ab. Patients with MS had higher AQP1-Ab levels than those with NMOsd (median 782.32 vs. $203.16 \mathrm{pg} / \mathrm{mL} ; p<0.001$ ). CLND5 levels were significantly higher in MS than in NMOsd patients (median $1.65 \mathrm{vs} .1 .00 \mathrm{ng} / \mathrm{mL}$; $p=0.004$ ). No statistically significant differences between MS and NMOsd were found for OCLN, VEGF and VE-Cadherin serum levels. In MS, AQ1-Ab levels were significantly lower in MS patients treated with immunomodulatory drugs vs. the treatment-naive (median $712.46 \mathrm{pg} / \mathrm{mL}$ vs. $942.73 \mathrm{pg} / \mathrm{mL}$, respectively). There was a positive correlation between CLDN5 and OCLN in both the MS and the NMOsd groups.
\end{abstract}

Conclusions and clinical implications. There is a different BBB disruption profile in MS and NMOsd, reflected by significantly higher CLDN5 and AQP1-Ab levels in MS samples. AQP1-Ab can be considered as a promising indicator of BBB disruption possibly associated with astrocytopathy.

Key words: neuromyelitis optica spectrum disorders, multiple sclerosis, anti-aquaporin 4 antibodies, anti-aquaporin 1 antibodies, cell-cell adhesion molecules

(Neurol Neurochir Pol 2022; 56 (4): 308-317)

Address for correspondence: Alicja Kalinowska-Łyszczarz, Department of Neurochemistry and Neuropathology, Poznan University of Medical Sciences, Przybyszewskiego 49 Str., 60-355 Poznan, Poland; e-mail: akalinowskalyszczarz@ump.edu.pl

Received: 02.11.2021 Accepted: 03.12.2021 Early publication date: 14.01 .2022

This article is available in open access under Creative Common Attribution-Non-Commercial-No Derivatives 4.0 International (CC BY-NC-ND 4.0) license, allowing to download articles and share them with others as long as they credit the authors and the publisher, but without permission to change them in any way or use them commercially. 


\section{Introduction}

Multiple sclerosis (MS) and neuromyelitis optica spectrum disorders (NMOsd) are autoimmune diseases of the central nervous system (CNS) which lead to demyelination in the brain and in the spinal cord $[1,2]$. Early and accurate distinction between these diseases is essential due to different prognosis and treatment strategies, but still remains challenging, despite typical clinical features, laboratory and magnetic resonance imaging (MRI) findings [2,3]. Until now, no specific marker for MS has been found [4]. Although the presence of oligoclonal bands in the cerebrospinal fluid (CSF) is very typical for MS, it is unspecific, because they can also be found in other diseases with white matter lesions, including NMOsd (15-30\% of cases) [5]. In turn, antibodies against aquaporin 4 ( $\mathrm{AQP} 4-\mathrm{IgG}$ ) are regarded as a specific marker for NMOsd, an essential element of the disease pathogenesis and a predictive factor $[2,6,7]$. Nevertheless, $10-25 \%$ of NMOsd patients are AQP4-IgG seronegative, and recent studies have shown that they could be seropositive for antibodies against myelin oligodendrocyte glycoprotein (MOG-Ab) $[5,8,9]$.

Aquaporin $1(\mathrm{AQP} 1)$ is a water channel expressed in the CNS epithelial cells of the choroid plexus, in the pia and the ependyma as well as in the microvascular endothelium outside the CNS but not in the cerebral endothelium $[10,11]$. Both $\mathrm{AQP1}$ and AQP4 may be selectively lost around active NMO lesions [12]. Serum anti-aquaporin 1 antibodies (AQP1-Ab) were found in AQP4-IgG seronegative and seropositive NMOsd but also in MS patients with spinal cord lesions (at low titres) [8]. Tzartos et al. [8] observed that AQP1-Ab in NMOsd belonged to the complement-activating IgG1 subclass and joined AQP1 extracellular domain, pointing to the conclusion that AQP1-Ab could be a new biomarker of NMOsd and play a role in disease pathogenesis. Nevertheless, this hypothesis has been challenged by further studies $[8,12,13]$.

Antibodies against myelin oligodendrocyte glycoprotein (MOG-Ab) could be present in patients with AQP4-IgG seronegative NMOsd as well as other CNS disorders, including recurrent optic neuritis $(\mathrm{ON})$, transverse myelitis (TM), paediatric acute disseminated encephalomyelitis (ADEM), and atypical MS (at low titres) $[9,14,15]$. The function of myelin oligodendrocyte glycoprotein (MOG), which is expressed on the outer layer of myelin sheaths and oligodendrocytes' processes, remains unclear, as well as whether MOG-Ab are pathogenic or secondary to a previous inflammation [14]. Nevertheless, MOG-Ab has become an important biomarker in demyelinating CNS diseases and an increasing number of cases seropositive for MOG-Ab indicates that another disorder probably exists. This has been named MOG antibody-associated disease (MOG-AD) [16].

Although NMOsd and MS are disorders with different immunopathogeneses, a common and fundamental event is early blood-brain barrier (BBB) disruption $[17,18]$. The junctions between endothelial cells are responsible for cell-cell adhesion, the maintenance of endothelial integrity, and providing a protective barrier which limits the exchange of molecules between the CNS and blood. Endothelial cells are connected through adherens and tight junctions (AJs and TJs) [19]. Because of that, BBB disruption can be explored by the measurement of serum levels of molecules that participate in the formation of the above-mentioned connections [20].

Cadherins, which can be found in AJs at the basolateral cell surface, are essential for formation and maintenance of intercellular junctions as well as for cell recognition, migration and communication [19,21,22]. Vascular-endothelial cadher in (VE-Cadherin), found in endothelial cells, has a function in intercellular adhesion, vascular integrity and permeability $[23,24]$. Importantly, cadherins play a role in the pathogenesis of various diseases, including cancers and inflammatory processes (particularly VE-Cadherin) [23, 25].

Claudin and occludin are transmembrane proteins present in TJs, which are located at the apical regions of the cells $[19,26]$. Claudins are responsible for regulation of epithelial permeability for ions and small molecules and interact with occludin, which plays a role in stabilisation and optimal barrier function of TJs [26, 27]. Claudin-5 (CLDN5) is present in endothelial cells in many tissues with different expression, but is particularly high in CNS vessels [28]. The expression of CLDN5 is regulated by various factors/events, e.g. vascular endothelial growth factor (VEGF) (decreased expression), matrix metalloproteinase (MMP) (degradation) as well as VE-Cadherin or glucocorticoids (increased expression) [28]. Dysregulation of TJs has been observed in many diseases, e.g. cancer, stroke, and inflammation [26]. It has been shown that inflammation led to increased internalisation of claudin and higher BBB permeability [29]. In turn, downregulation of occludin due to various factors, e.g. VEGF-induced occludin phosphorylation and ubiquitination, results in increased endothelial permeability [30]. Moreover, it has been shown that CLDN5 mediates leukocyte transendothelial migration in mice with experimental autoimmune encephalomyelitis (EAE) [31].

Vascular endothelial growth factor is a stimulator of angiogenesis, endothelial proliferation and permeability of microvessels [32]. It has been shown that VEGF led to lower expression of VE-cadherin and occludin in intercellular endothelial junctions and increased BBB permeability [32]. In patients with MS relapse, higher serum VEGF levels than healthy controls were observed [33]. Shimizu et al. [34] in turn found that the sera from AQP4-seropositive NMOsd patients reduced claudin- 5 in TJs in human brain microvascular endothelial cells, which resulted in BBB disruption and was reversed after anti-VEGF antibody administration.

\section{Clinical rationale for the study}

In our study we have assessed AQP4-IgG, AQP1-Ab, cellcell adhesion molecules, including claudin-5, occludin and VE-Cadherin, and VEGF in the sera of NMOsd and MS patients. 
We further explored the relationship between the anti-aquaporin antibodies' profile and BBB permeability measured by serum levels of the molecules that participate in intercellular junction formation in NMOsd and MS patients. We speculated that the potential differences between the results in the evaluated groups could provide new information about pathogenesis, and could be helpful in differential diagnosis between these diseases.

\section{Material and methods}

\section{Patients}

A total of 79 patients were recruited at the Department of Neurology, Heliodor Swiecicki University Hospital in Poznan, between June 2014 and August 2017. Twenty patients with NMOsd diagnosed according to the International Consensus Diagnostic Criteria for NMOsd [35] were incorporated into the study from those hospitalised in the Department of Neurology or screened for AQP4-IgG in the Department of Neurochemistry and Neuropathology by Poznan University of Medical Sciences. All NMOsd patients were initially seropositive for AQP4-IgG. Fifty-nine patients with RRMS diagnosed according to the $2010 \mathrm{McDonald}$ criteria [36] were recruited when evaluated or treated in the Department of Neurology or at the MS clinic. On study inclusion, all patients were clinically stable. Ten NMOsd patients were treated with oral steroids and/ or azathioprine: one with glatiramer acetate (GA), and nine were untreated (Supplementary Tab. 1). Most RRMS patients received platform injectable therapies, namely interferon-beta (IFN- $\beta$ ) or GA (33 and three patients, respectively), but 23 patients were not under immunomodulatory treatment at the moment of the evaluation (Supplementary Tab. 1).

The study protocol was approved by the Internal Review Ethics Board of Poznan University of Medical Sciences. All patients gave written consent to study participation.

\section{Laboratory analyses}

Serum samples were obtained from the study subjects and then kept frozen at $-80^{\circ} \mathrm{C}$ until further analysis. Screening for AQP4-IgG and MOG-Ab was performed using commercially available cell-based assays (CBA) according to the manufacturer's instructions (IIFT: NMOSD Screen 1, EUROIMMUN Medizinische Labordiagnostika AG, Germany). Serum levels of AQP1-Ab, VEGF and VE-Cadherin were determined with the use of ELISA kits, according to the manufacturer's instructions [Human AQP1 (Aquaporin-1) ELISA Kit, MyBioSource Inc., USA, BioVendor Laboratory Medicine Inc. Czech Republic and R\&D Systems Inc. Minneapolis, MN, USA]. The levels of AQP1-Ab and VE-Cadherin were expressed in picograms per millilitre $(\mathrm{pg} / \mathrm{mL})$ and VEGF in nanograms per millilitre (ng/mL).

To measure occludin (OCLN) and claudin (CLDN5) serum levels, we employed home-made ELISA tests elaborated at the Department of Neurochemistry and Neuropathology at Poznan University of Medical Sciences in Poland.
For OCLN ELISA testing, 96-well microplates (Immuno MaxiSorp Microplate, Thermo Scientific ${ }^{\mathrm{Tm}} \mathrm{Nunc}^{\mathrm{Tm}}$ ) were coated with mouse anti-occludin antibodies at a concentration of $1.0 \mu \mathrm{g} / \mathrm{mL}$ (Invitrogen, USA). After overnight incubation at $+4^{\circ} \mathrm{C}$, the plates were washed three times with phosphate-buffered salt (PBS) with $0.05 \%$ Tween 20 . Nonspecific binding sites were blocked with $3 \%$ bovine serum albumin (BSA) solution in PBS, and the plates were incubated for one hour and washed three times with PBS-0.05\% Tween20. Then, serial dilutions of the OCLN standard (Abnova) were prepared in the range from 2.75 to $0.043 \mathrm{ng} / \mathrm{mL}$. The OCLN standard and patients' sera were added to the particular holes. The plates were incubated for two hours and washed four times with PBS-0.05\% Tween 20 . Rabbit anti-occludin antibodies (concentration: $0.5 \mu \mathrm{g} / \mathrm{mL}$ ) (Invitrogen, USA) were added and the plates were incubated for two hours and washed four times with PBS- $0.05 \%$ Tween 20 . Goat anti-rabbit IgG conjugated with peroxidase $(66.7 \mathrm{ng} / \mathrm{mL})$ (Invitrogen, USA) was added and the plates were incubated for two hours and washed four times with PBS-0.05\% Tween 20 . Substrate Reagents R\&D were mixed in the ratio of $1: 1$ and added to the plates for colour reaction. After incubation in the darkness for 30 minutes, $2 \mathrm{~N}$ sulfuric acid was used as an inhibitor. After 5 minutes, absorbance was measured at wavelengths $450 \mathrm{~nm}$ and $540 \mathrm{~nm}$ using an ELx800 reader (BioTek, USA). The OCLN concentrations were read from the calibration curve (4-PL), which was determined with KC-Junior software (BioTek, USA). The concentrations of OCLN were expressed in nanograms per millilitre $(\mathrm{ng} / \mathrm{mL})$.

For CLDN5 ELISA testing, 96-well microplates (Immuno MaxiSorp Microplate, Thermo Scientific ${ }^{\mathrm{Tt}} \mathrm{Nunc}^{\mathrm{Tm}}$ ) were coated with mouse anti-claudin antibodies at concentration $0.5 \mathrm{mg} /$ $/ \mathrm{mL}$ (Invitrogen, USA). After overnight incubation at $+4^{\circ} \mathrm{C}$ and washing three times with washing buffer (PBS, EDTA and $0.05 \%$ Tween 20 ), a $2 \%$ solution of milk in PBS was added for blocking nonspecific binding sites. The plates were incubated for one hour and washed with washing buffer three times. Serial dilutions of the CLDN5 standard (Abnova) were prepared in the range from 16.0 to $0.25 \mathrm{ng} / \mathrm{mL}$. CLDN5 standard and examined patients' sera were added to the plate's holes with successive incubation for two hours and washed four times. Rabbit anti-human claudin antibodies $(0.25 \mu \mathrm{g} / \mathrm{mL})$ (Invitrogen, USA) were added and the plates were incubated for two hours and washed with washing buffer four times. Goat anti-rabbit IgG with peroxidase $(66.7 \mathrm{ng} / \mathrm{mL})$ (Invitrogen, USA) was added and the plates were incubated for two hours and washed four times with washing buffer. Substrate Reagents R\&D were added to the plates for colour reaction. This was followed by incubation in the darkness for 30 minutes and the use of $2 \mathrm{~N}$ sulfuric acid as an inhibitor. After 5 minutes, absorbance was measured at wavelengths $450 \mathrm{~nm}$ and $540 \mathrm{~nm}$ with an ELx800 reader (BioTek, USA). The concentrations of CLDN5 were read from the calibration curve (4-PL), which was created with KC-Junior software (BioTek, USA). The concentrations of CLDN5 were expressed in nanograms per millilitre $(\mathrm{ng} / \mathrm{mL})$. 


\section{Statistical analyses}

Statistical analyses were performed with the use of R 4.0.3 [R Core Team (2020). R: A language and environment for statistical computing. R Foundation for Statistical Computing, Vienna, Austria. URL https://www.R-project.org].

Mann-Whitney test was used to compare quantitative variables between the two groups. Relationship between two quantitative variables was assessed with Spearman's coefficient of correlation. Significance level for all statistical tests was set at 0.05 .

\section{Results}

\section{Baseline}

Seventy-nine patients were included in our study: 20 with NMOsd (19 women, one man) and 59 with MS (46 women, 13 men). Females formed the large majority in both groups (77.97\% in MS and 95\% in NMOsd; $\mathrm{p}=0.102$ ). The evaluated groups differed in basic clinical characteristics, as NMOsd patients compared to those with MS were older (median age $42.5 v$ s. 35 years), had a greater disease duration (median $8 v s$. 3 years), and a higher EDSS score (median $3.75 v s .1 .0$ ) (Fig. 1A-C, Tab. 1).

\section{Antibodies, VEGF and intercellular adhesion molecules in NMOsd and MS groups}

Patients with MS had higher AQP1-Ab and CLDN5 levels than those with NMOsd (median 782.32 vs. $203.16 \mathrm{pg} / \mathrm{mL}$; $\mathrm{p}<0.001$, for AQP1-Ab and $1.65 v s .1 .00 \mathrm{ng} / \mathrm{mL} ; \mathrm{p}=0.004$, for CLDN5) (Fig. 1D-E). AQP4-IgG appeared only in NMOsd subjects (6/20;30\%). AQP1-Ablevelswerehigher in NMOsd cases seropositive than seronegative for AQP4-IgG, but the difference was statistically insignificant (315.27 vs. $163.82 \mathrm{pg} / \mathrm{mL}$, respectively) (Supplementary Tab. 2, Supplementary Figure 1). All MS and NMOsd patients were seronegative for MOG-Ab.

No statistically significant differences between MS and NMOsd groups were found for VEGF (median $250.08 v s$. $248.78 \mathrm{ng} / \mathrm{mL} ; \mathrm{p}=0.918$, respectively), VE-Cadherin (median $5,090.19 v$ s. $4,490.1 \mathrm{pg} / \mathrm{mL} ; \mathrm{p}=0.08$, respectively) or OCLN (median 0.58 vs. $0.42 \mathrm{ng} / \mathrm{mL} ; \mathrm{p}=0.1$, respectively) (Fig. 1G-F).

$\mathrm{AQ1}-\mathrm{Ab}$ levels differed within the MS group, as the median was $712.46 \mathrm{pg} / \mathrm{mL}$ in patients who received immunomodulatory treatment versus $942.73 \mathrm{pg} / \mathrm{mL}$ in those who were untreated $(p=0.044)$. There were no statistically significant differences between the treated and untreated NMOsd patients as well as for other parameters within MS group.

The above results are set out in Table 1.

\section{Correlations}

Sex and age were not associated with the evaluated parameters' levels in the cohort.

Disease duration correlated positively with CLDN5, but no other molecules' levels, in NMOsd patients $(\mathrm{r}=0.513$; $\mathrm{p}=0.021)($ Fig. $2 \mathrm{~A})$. In the MS group, the relationship between disease duration and the evaluated molecules did not reach statistical significance.

In MS, EDSS score correlated positively with VE-Cadherin $(\mathrm{r}=0.464 ; \mathrm{p}=0.009)$ and AQP1-Ab $(\mathrm{r}=0.328 ; \mathrm{p}=0.019)$ and negatively with OCLN $(r=-0.298 ; p=0.042)$, while in NMOsd, correlations between EDSS and the tested molecules levels were not significant (Fig. 2B-D).

CLDN5 and OCLN were positively correlated in the MS $(\mathrm{r}=0.599 ; \mathrm{p}<0.001)$ and the NMOsd $(\mathrm{r}=0.74 ; \mathrm{p}<0.001)$ groups (Supplementary Figures 2-3). There was a positive, but statistically insignificant, correlation between $\mathrm{AQP} 1-\mathrm{Ab}$ and CLDN5 in MS patients $(r=0.234 ; \mathrm{p}=0.098)$.

The correlation results are set out in Table 2 .

\section{Discussion}

The most surprising finding of our study was that MS patients had significantly higher AQP1-Ab levels compared to those with NMOsd.

Moreover, AQP1-Ab levels showed a trend for being higher in $\mathrm{AQP} 4-\mathrm{IgG}$ seropositive than in $\mathrm{AQP} 4-\mathrm{IgG}$ seronegative NMOsd subjects. Previous studies showed that AQP1-Ab was present mainly in the sera of NMOsd patients (seropositive as well as seronegative for $\mathrm{AQP4}$-IgG), but also in some MS patients [8, 37, 38]. Garcia-Miranda et al. [39] evaluated the sera of patients with NMOsd, MS, idiopathic ON or myelitis, clinically isolated brainstem syndrome, other diseases and healthy controls using a CBA and ELISA. They observed a lack of AQP1-Ab as well as MOG-IgG (except for one patient with idiopathic myelitis) in the whole cohort, and the presence of AQP4-IgG only in NMOsd patients' sera. Schanda et al. [13] also reported the absence of AQP1-Ab in the sera of all NMOsd and MS subjects, as assessed by a CBA. The inconsistent results of various studies indicate that there is a need for further exploration of the AQP1-Ab in patients with demyelinating diseases. Interestingly, higher AQP1-Ab levels were found in immunopathological pattern III of demyelination, which suggests a relation to astrocytic damage [40]. Thus, AQP1-Ab may be associated with disruption at the level of $B B B$ rather than with primary immunological response. The correlation of AQP1-Ab with CLDN-5 that we observed in the MS group may lend support to such speculation.

All MS and NMOsd patients participating in the study were seronegative for MOG-Ab. This is in accordance with previous studies, which showed that MOG-Ab could be present in NMOsd patients seronegative for AQP4-IgG and with atypical MS [14].

AQP4-IgG were present in only 6/20 NMOsd patients, although originally all of them were seropositive. Nevertheless, it is known that $\mathrm{AQP} 4-\mathrm{IgG}$ serum titres can change depending on disease activity (higher in relapse than in remission) or treatment (decrease after/under steroids, azathioprine, cyclophosphamide or rituximab) [41]. This may explain seronegativity for AQP4-IgG in some NMOsd cases, as 6/14 patients 


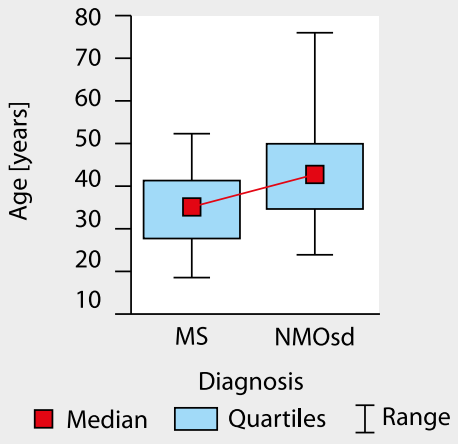

C

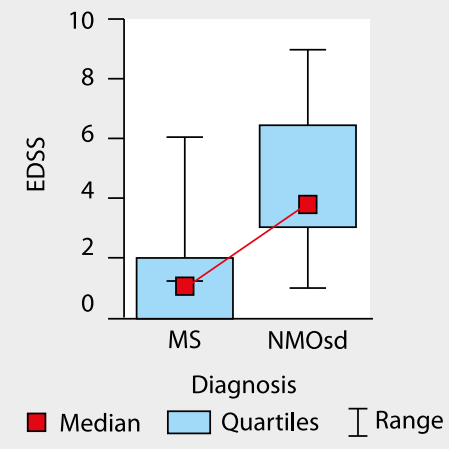

E

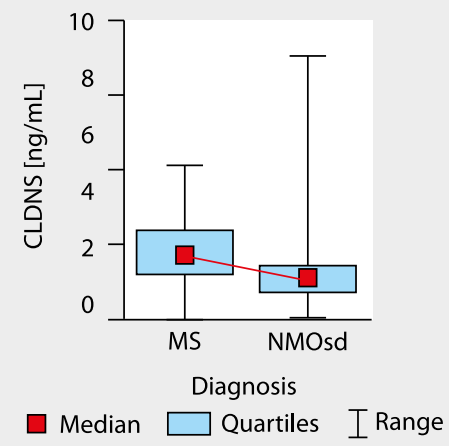

G

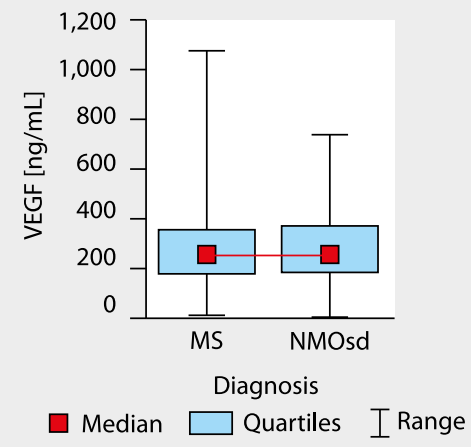

B

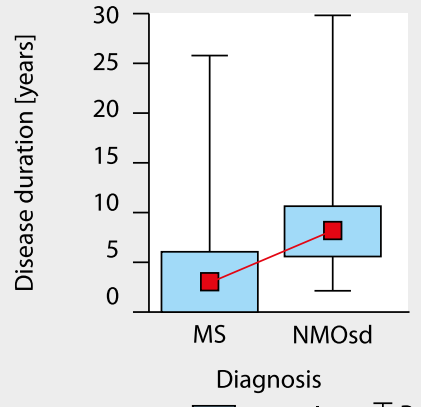

$\square$ Median $\square$ Quartiles I Range

D

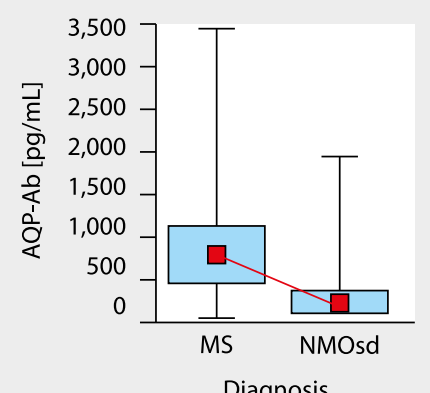

$\square$ Median $\square$ Quartiles I Range

$\mathbf{F}$

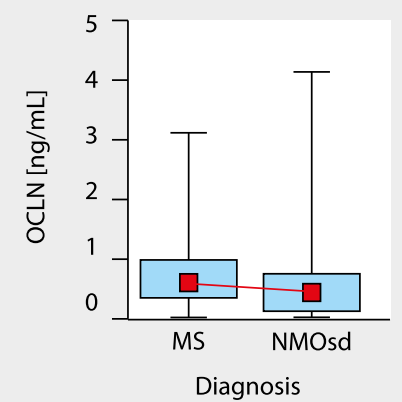

$\square$ Median $\square$ Quartiles I Range

H

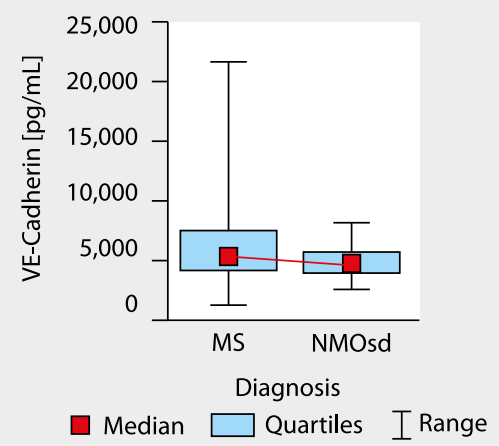

Figure 1. Comparison of evaluated parameters in MS and NMOsd groups: age (A), disease duration (B), EDSS (C), AQP1-Ab (D), CLDN5 (E), OCLN (F), VEGF (G), VE-Cadherin (H); EDSS - Kurtzke Expanded Disability Status Scale; MS - multiple sclerosis; NMOsd - neuromyelitis optica spectrum disorders 
Table 1. Clinical characteristics of evaluated groups and results of laboratory parameters

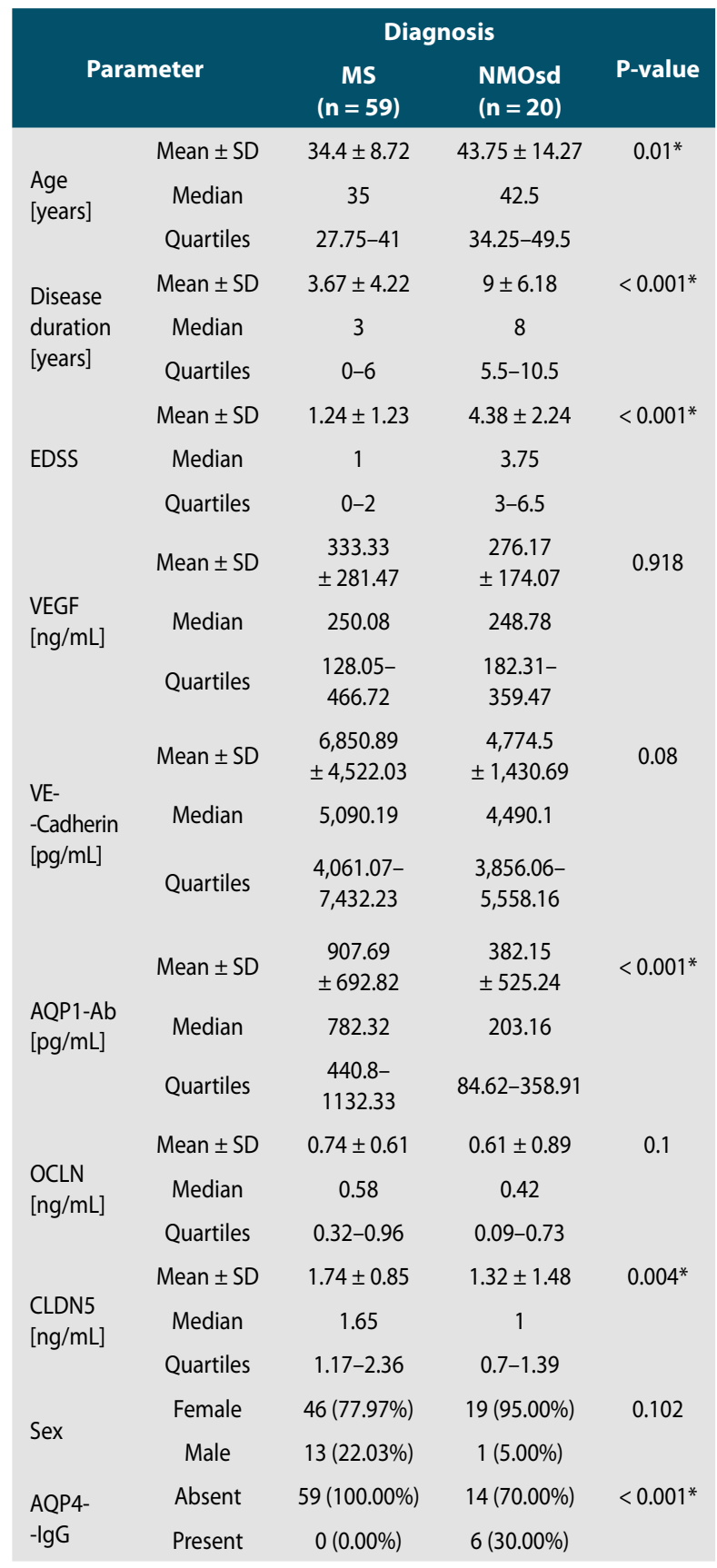

AQP1-Ab - anti-aquaporin 1 antibodies; AQP4-IgG — anti-aquaporin 4 antibodies; CLDN5 claudin-5; EDSS - Kurtzke Expanded Disability Status Scale; MS - multiple sclerosis; NMOsd - neuromyelitis optica spectrum disorders; OCLN — occludin; SD — standard deviation;VEGF vascular endothelial growth factor; $\mathrm{p}-$ Mann-Whitney test for quantitative variables, chi-squared or Fisher's exact test for qualitative variables; * ${ }^{*}$ statistically significant $(p<0.05)$

with the change in serostatus received steroids and/or other immunosuppression during the study. However, 8/14 AQP4IgG seronegative subjects were untreated and 5/6 patients who maintained AQP4-IgG seropositivity were under therapy, so in this subgroup other variables, e.g. subclinical disease activity, probably existed. Further studies in a larger NMOsd group are needed to evaluate factors influencing the change in AQP4-IgG serostatus. Whether the same paradigm applies to AQP1-Ab remains to be established. CLDN5 levels were significantly higher in NMOsd patients seronegative than seropositive for AQP4-IgG, but because of a small sample size, this observation should be interpreted with caution and assessed in a larger population. The absence of AQP4-IgG in MS patients' sera confirmed the generally understood high specificity (91\%) of AQP4-IgG for NMOsd [42].

In our study, CLND5 levels were significantly higher in the MS than in the NMOsd group. OCLN levels were also higher in MS, but the difference did not reach statistical significance. Mandel at al. showed that in MS patients during relapse claudin-1 and claudin-5 levels were increased in peripheral blood leukocytes (PBLs), which could facilitate leukocytes extravasation into the CNS. The claudins levels in PBLs decreased after glucocorticosteroids treatment, and pre-treatment claudin-5 mRNA levels were lower in patients with a good response to IFN- $\beta$ [43]. Interestingly, claudin- 5 is localised more externally in TJs at BBB than occludin, and thus it can be released more easily to the circulation [27]. This led to the conclusion that claudin- 5 could be a candidate biomarker of disease activity in MS.

Importantly, we observed a positive correlation between CLDN5 and OCLN in both the MS and the NMOsd groups. This could be explained by the fact that there is an interaction between claudin and occludin within TJs [44]. Moreover, in the disease several abnormalities in the expression of TJs' proteins have been reported, e.g. in MS the loss of CLDN5 and occludin resulted in impaired BBB function [45].

Our study also revealed a negative correlation between EDSS and OCLN in the MS group. Assuming that TJs' protein serum levels reflect the BBB disruption in inflammation, this could be explained by the fact that EDSS increases with disease duration and the intensity of inflammatory response differs in particular stages of demyelinating diseases. Specifically, in MS, inflammation dominates at the early, and neurodegeneration at the late, stages of the disease [46]. On the other hand, in NMOsd, where EDSS increases relatively early within the disease course, we observed that disease duration correlated positively with CLDN5 but not with occludin. Further studies on a larger cohort are necessary to verify whether these findings would be replicated.

We found that immunomodulatory treatment was associated with lower levels of AQP1-Ab in MS patients. We assume that AQP1-Ab could be a marker of BBB disruption. In turn, $\mathrm{BBB}$ permeability increases in inflammation, as mentioned before. It is known that IFN- $\beta$ and GA treatment moderate the inflammatory response [47]. IFN- $\beta$ exerts various anti-inflammatory and immunomodulatory effects, e.g. suppression of T-lymphocytes activation and proliferation, or reduction of proinflammatory cytokines' concentrations [48]. Furthermore, there is evidence for the stabilising effect of IFN- $\beta$ on BBB [47]. We speculate that inflammation decrease due to treatment led to improvement of $\mathrm{BBB}$ properties and 


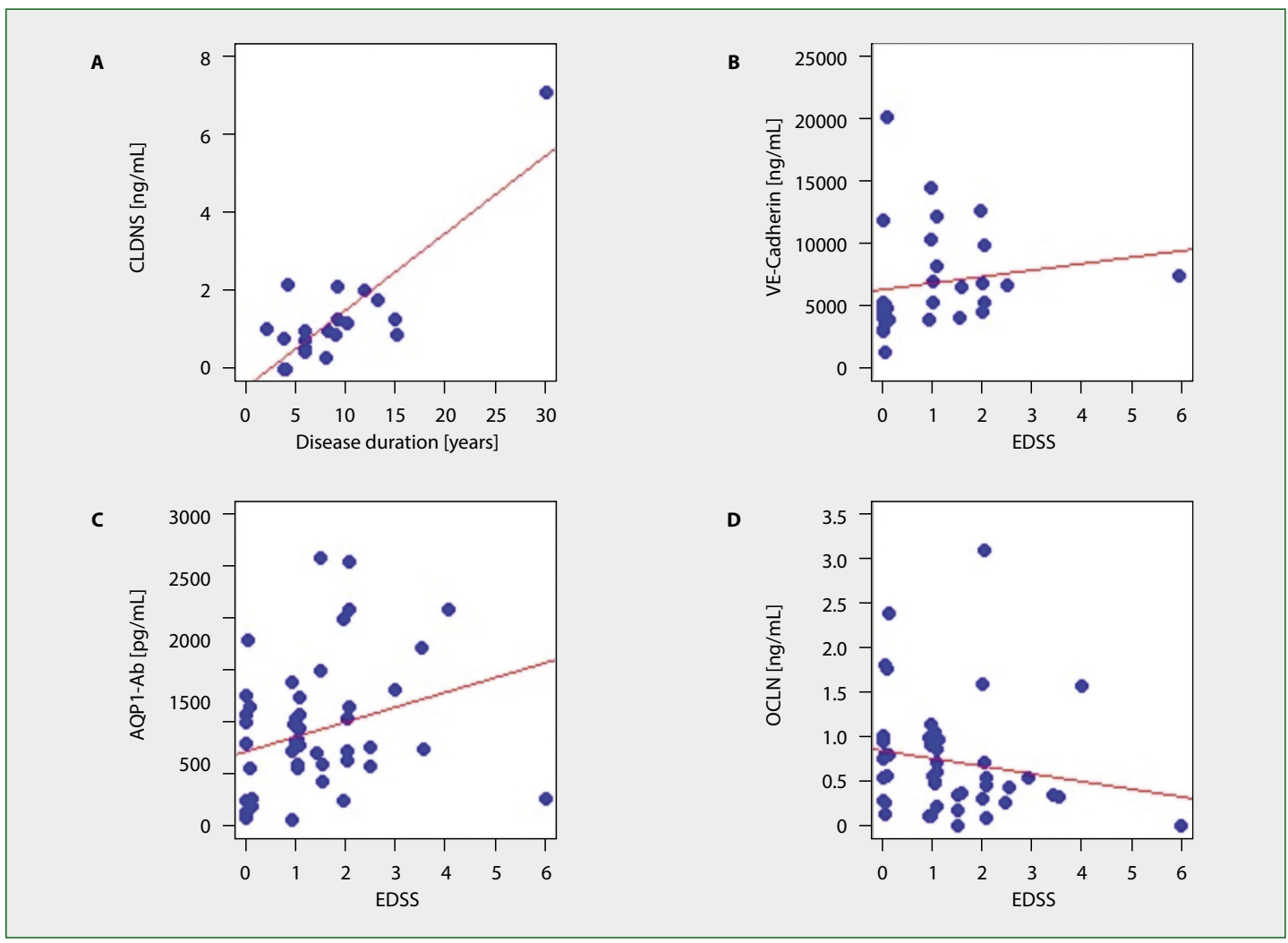

Figure 2. Correlations between: CLDN5 and disease duration in NMOsd group (A), VE-Cadherin and EDSS in MS group (B), AQP1-Ab and EDSS in MS group (C), OCLN and EDSS in MS group (D); EDSS - Kurtzke Expanded Disability Status Scale; MS - multiple sclerosis; NMOsd - neuromyelitis optica spectrum disorders

Table 2. Correlations between assessed parameters in MS and NMOsd groups

\begin{tabular}{|c|c|c|c|c|c|}
\hline \multicolumn{6}{|c|}{ MS } \\
\hline & VEGF & VE-Cadherin & AQP1-Ab & OCLN & CLDN5 \\
\hline VEGF & - & $\begin{array}{c}r=-0.021 \\
p=0.903\end{array}$ & $\begin{array}{l}r=0.172 \\
p=0.315\end{array}$ & $\begin{array}{l}r=0.099 \\
p=0.595\end{array}$ & $\begin{array}{l}r=0.154 \\
p=0.409\end{array}$ \\
\hline VE-Cadherin & $\begin{array}{c}r=-0.021 \\
p=0.903\end{array}$ & - & $\begin{array}{c}r=-0.039 \\
p=0.822\end{array}$ & $\begin{array}{c}r=-0.289 \\
p=0.115\end{array}$ & $\begin{array}{l}r=0.002 \\
p=0.991\end{array}$ \\
\hline$A Q P 1-A b$ & $\begin{array}{l}r=0.172 \\
p=0.315\end{array}$ & $\begin{array}{c}r=-0.039 \\
p=0.822\end{array}$ & - & $\begin{array}{l}r=0.108 \\
p=0.447\end{array}$ & $\begin{array}{l}r=0.234 \\
p=0.098\end{array}$ \\
\hline OCLN & $\begin{array}{l}r=0.099 \\
p=0.595\end{array}$ & $\begin{array}{c}r=-0.289 \\
p=0.115\end{array}$ & $\begin{array}{l}r=0.108 \\
p=0.447\end{array}$ & - & $\begin{array}{l}r=0.599 \\
p<0.001^{*}\end{array}$ \\
\hline CLDN5 & $\begin{array}{l}r=0.154 \\
p=0.409\end{array}$ & $\begin{array}{l}r=0.002 \\
p=0.991\end{array}$ & $\begin{array}{l}r=0.234 \\
p=0.098\end{array}$ & $\begin{array}{l}r=0.599 \\
p<0.001^{*}\end{array}$ & - \\
\hline \multicolumn{6}{|c|}{ NMOsd } \\
\hline & VEGF & VE-Cadherin & AQP1-Ab & OCLN & CLDN5 \\
\hline VEGF & - & $\begin{array}{l}r=-0.03 \\
p=0.905\end{array}$ & $\begin{array}{l}r=0.107 \\
p=0.663\end{array}$ & $\begin{array}{c}r=-0.167 \\
p=0.496\end{array}$ & $\begin{array}{c}r=-0.185 \\
p=0.448\end{array}$ \\
\hline VE-Cadherin & $\begin{array}{l}r=-0.03 \\
p=0.905\end{array}$ & - & $\begin{array}{c}r=-0.278 \\
p=0.249\end{array}$ & $\begin{array}{l}r=0.055 \\
p=0.824\end{array}$ & $\begin{array}{l}r=0.123 \\
p=0.616\end{array}$ \\
\hline$A Q P 1-A b$ & $\begin{array}{l}r=0.107 \\
p=0.663\end{array}$ & $\begin{array}{c}r=-0.278 \\
p=0.249\end{array}$ & - & $\begin{array}{c}r=-0.365 \\
p=0.113\end{array}$ & $\begin{array}{c}r=-0.108 \\
p=0.651\end{array}$ \\
\hline OCLN & $\begin{array}{c}r=-0.167 \\
p=0.496\end{array}$ & $\begin{array}{l}r=0.055 \\
p=0.824\end{array}$ & $\begin{array}{c}r=-0.365 \\
p=0.113\end{array}$ & - & $\begin{array}{c}r=0.74 \\
p<0.001^{*}\end{array}$ \\
\hline CLDN5 & $\begin{array}{c}r=-0.185 \\
p=0.448\end{array}$ & $\begin{array}{l}r=0.123 \\
p=0.616\end{array}$ & $\begin{array}{c}r=-0.108 \\
p=0.651\end{array}$ & $\begin{array}{c}r=0.74 \\
p<0.001^{*}\end{array}$ & - \\
\hline
\end{tabular}

AQP1-Ab - anti-aquaporin 1 antibodies; AQP4-IgG - anti-aquaporin 4 antibodies; CLDN5 - claudin-5; MS - multiple sclerosis; NMOsd - neuromyelitis optica spectrum disorders; OCLN - occludin; $r$ Spearman's correlation coefficient; VEGF — vascular endothelial growth factor; *statistically significant $(p<0.05)$ 
reduction of serum levels of molecules, which could be markers of its breakdown.

Our study has several limitations. Firstly, the number of NMOsd patients was relatively small to establish the exact profile of antibodies and molecules of intercellular junctions. Nevertheless, it should be noted that this was a single centre study and that NMOsd is a rare disease, particularly in Caucasians (in USA and Europe the prevalence is $0.5-10 / 100,000$, and the MS to NMOsd ratio around 50-100) [2, 49]. Given the rarity of NMOsd, with only few new cases diagnosed each year, and a three-year recruitment timeline, this limitation could not be overcome. Possibly due to the sample size, we were unable to identify the relationship between evaluated antibodies or molecules and various factors, e.g. disease activity (remission $v s$. relapse), EDSS score, MRI findings, concomitant diseases and treatment status. Furthermore, in our cohort there were untreated patients as well as patients on various therapies. As we recruited patients consecutively at the Department of Neurology, in some cases there was a lack of blood samples collected before treatment initiation, so we were unable to assess its potential influence on the evaluated parameters.

In the future, it would be interesting to compare antibodies' titres and intercellular junction molecules' levels in larger cohorts of MS and NMOsd patients receiving particular therapies. Moreover, we were not able to perform the CSF examination due to ethical reasons, as in most cases the lumbar puncture had already been done, and an additional lumbar puncture could cause potential side effects. Nonetheless, in the future it would be valuable to assess the relationship between cell-cell adhesion molecules, VEGF, AQP1-Ab, AQP4-IgG and the CSF parameters.

\section{Conclusions and future directions}

Based on the results of our study, we draw the following conclusions:

1. AQP1-Ab are not a specific marker for NMOsd, as in our cohort AQP1-Ab titres were even higher in the sera of MS than NMOsd patients;

2. there is a different BBB disruption profile in MS and NMOsd;

3. among the cell-cell adhesion molecules that we evaluated, claudin- 5 serum level reflected these differences best, as it was significantly higher in MS than NMOsd patients;

4. $\mathrm{AQP} 1-\mathrm{Ab}$ can be considered as a promising indicator of BBB disruption possibly associated with astrocytopathy. Further studies on a larger sample size are necessary to evaluate the exact profile of BBB disruption in NMOsd and MS patients expressed by increased serum levels of intercellular junctions' molecules and their dependence on antibodies serostatus or titres, as well as other factors including disease activity, MRI findings, concomitant diseases and treatment.
Acknowledgments: The authors wish to acknowledge Renata Gajek for technical assistance with blood sampling and laboratory procedures.

Ethics statement: The study was approved by the Internal Review Ethics Board at Poznan University of Medical Sciences. Conflict of interest: None.

Funding: This study was supported by National Science Centre grants 2012/07/B/NZ6/03529 and 2012/05/D/ NZ6/00989 (AKE).

\section{References}

1. Lassmann H, Brück W, Lucchinetti CF. The immunopathology of multiple sclerosis: an overview. Brain Pathol. 2007; 17(2): 210-218, doi: 10.1111/j.1750-3639.2007.00064.x, indexed in Pubmed: 17388952.

2. Wingerchuk DM. Neuromyelitis optica spectrum disorders. Continuum (Minneap Minn). 2010; 16(5 Multiple Sclerosis): 105-121, doi: 10.1212/01.con.0000389937.69413.15, indexed in Pubmed: 22810601.

3. Selmaj K, Selmaj I. Novel emerging treatments for NMOSD. Neurol Neurochir Pol. 2019; 53(5): 317-326, doi: 10.5603/PJNNS. a2019.0049, indexed in Pubmed: 31664710.

4. Sapko K, Jamroz-Wiśniewska A, Marciniec M, et al. Biomarkers in Multiple Sclerosis: a review of diagnostic and prognostic factors. Neurol Neurochir Pol. 2020; 54(3): 252-258, doi: 10.5603/PJNNS. a2020.0037, indexed in Pubmed: 32462652.

5. Wingerchuk DM, Lennon V, Lucchinetti C, et al. The spectrum of neuromyelitis optica. Lancet Neurol. 2007; 6(9): 805-815, doi: 10.1016/ s1474-4422(07)70216-8, indexed in Pubmed: 17706564.

6. Lennon VA, Kryzer TJ, Pittock SJ, et al. IgG marker of optic-spinal multiple sclerosis binds to the aquaporin-4 water channel. J Exp Med. 2005; 202(4): 473-477, doi: 10.1084/jem.20050304, indexed in Pubmed: 16087714.

7. Weinshenker BG, Wingerchuk DM, Vukusic S, et al. Neuromyelitis optica IgG predicts relapse after longitudinally extensive transverse myelitis. Ann Neurol. 2006; 59(3): 566-569, doi: 10.1002/ana.20770, indexed in Pubmed: 16453327.

8. Tzartos JS, Stergiou C, Kilidireas K, et al. Anti-aquaporin-1 autoantibodies in patients with neuromyelitis optica spectrum disorders. PLoS One. 2013; 8(9): e74773, doi: 10.1371/journal.pone.0074773, indexed in Pubmed: 24086369.

9. Saadoun S, Waters P, Owens GP, et al. Neuromyelitis optica MOG-IgG causes reversible lesions in mouse brain. Acta Neuropathol Commun. 2014; 2: 35, doi: 10.1186/2051-5960-2-35, indexed in Pubmed: 24685353.

10. Bonomini F, Francesca B, Rezzani R. Aquaporin and blood brain barrier. Curr Neuropharmacol. 2010; 8(2): 92-96, doi: 10.2174/157015910791233132, indexed in Pubmed: 21119879.

11. Hirt L, Price M, Benakis C, et al. Aquaporins in neurological disorders. Clin Transl Neurosci. 2018; 2(1): 2514183X1775290, doi: $10.1177 / 2514183 \times 17752902$.

12. Misu T, Höftberger R, Fujihara $K$, et al. Presence of six different lesion types suggests diverse mechanisms of tissue injury in neuromyelitis optica. Acta Neuropathol. 2013; 125(6): 815827, doi: 10.1007/s00401-013-1116-7, indexed in Pubmed: 23579868. 
13. Schanda $\mathrm{K}$, Waters $\mathrm{P}$, Holzer $\mathrm{H}$, et al. Antibodies to aquaporin-1 are not present in neuromyelitis optica. Neurol Neuroimmunol Neuroinflamm. 2015; 2(6): e160, doi: 10.1212/NXI.0000000000000160, indexed in Pubmed: 26468473.

14. Peschl P, Bradl M, Höftberger R, et al. Myelin oligodendrocyte glycoprotein: deciphering a target in inflammatory demyelinating diseases. Front Immunol. 2017; 8: 529, doi: 10.3389/fimmu.2017.00529, indexed in Pubmed: 28533781.

15. Mader S, Gredler V, Schanda K, et al. Complement activating antibodies to myelin oligodendrocyte glycoprotein in neuromyelitis optica and related disorders. J Neuroinflammation. 2011; 8: 184, doi: 10.1186/1742-2094-8-184, indexed in Pubmed: 22204662.

16. Ambrosius W, Michalak S, Kozubski W, et al. Myelin oligodendrocyte glycoprotein antibody-associated disease: current insights into the disease pathophysiology, diagnosis and management. Int J Mol Sci. 2020; 22(1): 100, doi: 10.3390/ijms22010100, indexed in Pubmed: 33374173.

17. Minagar A, Alexander JS. Blood-brain barrier disruption in multiple sclerosis. Mult Scler. 2003; 9(6): 540-549, doi: 10.1191/1352458503ms965oa, indexed in Pubmed: 14664465.

18. Asgari N, Flanagan EP, Fujihara K, et al. Disruption of the leptomeningeal blood barrier in neuromyelitis optica spectrum disorder. Neurol Neuroimmunol Neuroinflamm. 2017; 4(4): e343, doi: 10.1212/NXI.0000000000000343, indexed in Pubmed: 28451627.

19. Hartsock A, Nelson WJ. Adherens and tight junctions: structure, function and connections to the actin cytoskeleton. Biochim Biophys Acta. 2008; 1778(3): 660-669, doi: 10.1016/j.bbamem.2007.07.012, indexed in Pubmed: 17854762.

20. Olsson A, Gustavsen S, Langkilde AR, et al. Circulating levels of tight junction proteins in multiple sclerosis: association with inflammation and disease activity before and after disease modifying therapy. Mult Scler Relat Disord. 2021; 54: 103136, doi: 10.1016/j. msard.2021.103136, indexed in Pubmed: 34247104.

21. Albelda SM, Buck CA. Integrins and other cell adhesion molecules. FASEB J. 1990; 4(11): 2868-2880, doi: 10.1096/fasebj.4.11.2199285, indexed in Pubmed: 2199285.

22. Angst BD, Marcozzi C, Magee Al. The cadherin superfamily: diversity in form and function. J Cell Sci. 2001; 114(Pt 4): 629-641, indexed in Pubmed: 11171368.

23. Vestweber D. Cadherins in tissue architecture and disease. J Mol Med (Berl). 2015; 93(1): 5-11, doi: 10.1007/s00109-014-1231-5, indexed in Pubmed: 25488198.

24. Dejana E. Endothelial adherens junctions: implications in the control of vascular permeability and angiogenesis. J Clin Invest. 1996; 98(9): 1949-1953, doi: 10.1172/JCl118997, indexed in Pubmed: 8903311.

25. Yu W, Yang Li, Li T, et al. Cadherin signaling in cancer: its functions and role as a therapeutic target. Front Oncol. 2019; 9: 989, doi: 10.3389/ fonc.2019.00989, indexed in Pubmed: 31637214.

26. Cummins PM. Occludin: one protein, many forms. Mol Cell Biol. 2012; 32(2): 242-250, doi: 10.1128/MCB.06029-11, indexed in Pubmed: 22083955.

27. Findley MK, Koval M. Regulation and roles for claudin-family tight junction proteins. IUBMB Life. 2009; 61(4): 431-437, doi: 10.1002/ iub.175, indexed in Pubmed: 19319969.

28. Greene C, Hanley N, Campbell M. Claudin-5: gatekeeper of neurological function. Fluids Barriers CNS. 2019; 16(1): 3, doi: 10.1186/ s12987-019-0123-z, indexed in Pubmed: 30691500.
29. Walsh S. Modulation of tight junction structure and function by cytokines. Adv Drug Deliv Rev. 2000; 41(3): 303-313, doi: 10.1016/ s0169-409x(00)00048-x, indexed in Pubmed: 10854688.

30. Murakami T, Felinski EA, Antonetti DA. Occludin phosphorylation and ubiquitination regulate tight junction trafficking and vascular endothelial growth factor-induced permeability. J Biol Chem. 2009; 284(31): 2103621046, doi: 10.1074/jbc.M109.016766, indexed in Pubmed: 19478092.

31. Paul D, Baena V, Ge S, et al. Appearance of claudin-5 leukocytes in the central nervous system during neuroinflammation: a novel role for endothelial-derived extracellular vesicles. J Neuroinflammation. 2016; 13(1): 292, doi: 10.1186/s12974-016-0755-8, indexed in Pubmed: 27852330.

32. Kevil CG, Payne DK, Mire E, et al. Vascular permeability factor/vascular endothelial cell growth factor-mediated permeability occurs through disorganization of endothelial junctional proteins. J Biol Chem. 1998; 273(24): 15099-15103, doi: 10.1074/jbc.273.24.15099, indexed in Pubmed: 9614120.

33. Melamed E, Levy M, Waters PJ, et al. Update on biomarkers in neuromyelitis optica. Neurol Neuroimmunol Neuroinflamm. 2015; 2(4): e134, doi: 10.1212/NXI.0000000000000134, indexed in Pubmed: 26236760.

34. Shimizu F, Sano Y, Takahashi T, et al. Sera from neuromyelitis optica patients disrupt the blood-brain barrier. J Neurol Neurosurg Psychiatry. 2012; 83(3): 288-297, doi: 10.1136/jnnp-2011-300434, indexed in Pubmed: 22100760.

35. Wingerchuk DM, Banwell B, Bennett JL, et al. International Panel for NMO Diagnosis. International consensus diagnostic criteria for neuromyelitis optica spectrum disorders. Neurology. 2015; 85(2): 177-189, doi: 10.1212/WNL.0000000000001729, indexed in Pubmed: 26092914.

36. Polman $\mathrm{CH}$, Reingold SC, Banwell B, et al. Diagnostic criteria for multiple sclerosis: 2010 revisions to the McDonald criteria. Ann Neurol. 2011; 69(2): 292-302, doi: 10.1002/ana.22366, indexed in Pubmed: 21387374.

37. Long Y, Zheng Y, Shan F, et al. Development of a cell-based assay for the detection of anti-aquaporin 1 antibodies in neuromyelitis optica spectrum disorders. J Neuroimmunol. 2014; 273(1-2): 103-110, doi: 10.1016/j.jneuroim.2014.06.003, indexed in Pubmed: 24972850.

38. Tüzün E, Tzartos J, Ekizoğlu E, et al. Aquaporin-1 antibody in neuromyelitis optical patients. Eur Neurol. 2014; 72(5-6): 271-272, doi: 10.1159/000364904, indexed in Pubmed: 25277962.

39. García-Miranda P, Morón-Civanto FJ, Martínez-Olivo MD, et al. Predictive value of serum antibodies and point mutations of AQP4, AQP1 and MOG in a cohort of spanish patients with neuromyelitis optica spectrum disorders. Int J Mol Sci. 2019; 20(22): 5810, doi: 10.3390/ ijms20225810, indexed in Pubmed: 31752329.

40. Stork L, Ellenberger D, Ruprecht K, et al. Antibody signatures in patients with histopathologically defined multiple sclerosis patterns. Acta Neuropathol. 2020; 139(3): 547-564, doi: 10.1007/s00401019-02120-x, indexed in Pubmed: 31950335.

41. Jarius $S$, Aboul-Enein F, Waters P, et al. Antibody to aquaporin-4 in the long-term course of neuromyelitis optica. Brain. 2008; 131(Pt 11): 3072 3080, doi: 10.1093/brain/awn240, indexed in Pubmed: 18945724.

42. Lennon VA, Wingerchuk DM, Kryzer TJ, et al. A serum autoantibody marker of neuromyelitis optica: distinction from multiple sclerosis. Lancet. 2004; 364(9451): 2106-2112, doi: 10.1016/S0140-6736(04)17551-X, indexed in Pubmed: 15589308.

43. Mandel I, Paperna T, Glass-Marmor L, et al. Tight junction proteins expression and modulation in immune cells and multiple sclerosis. J Cell Mol Med. 2012; 16(4): 765-775, doi: 10.1111/j.1582-4934.2011.01380.x, indexed in Pubmed: 21762372. 
44. Shen L, Weber CR, Turner JR. The tight junction protein complex undergoes rapid and continuous molecular remodeling at steady state. J Cell Biol. 2008; 181(4): 683-695, doi: 10.1083/jcb.200711165, indexed in Pubmed: 18474622.

45. Alvarez JI, Cayrol R, Prat A. Disruption of central nervous system barriers in multiple sclerosis. Biochim Biophys Acta. 2011; 1812(2): 252-264, doi: 10.1016/j.bbadis.2010.06.017, indexed in Pubmed: 20619340.

46. Lassmann H. Pathology and disease mechanisms in different stages of multiple sclerosis. J Neurol Sci. 2013; 333(1-2): 1-4, doi: 10.1016/j.jns.2013.05.010, indexed in Pubmed: 23735777.
47. Balasa R, Barcutean L, Mosora O, et al. Reviewing the significance of blood-brain barrier disruption in multiple sclerosis pathology and treatment. Int J Mol Sci. 2021; 22(16): 8370, doi: 10.3390/ ijms22168370, indexed in Pubmed: 34445097.

48. Dhib-Jalbut S, Marks S. Interferon-beta mechanisms of action in multiple sclerosis. Neurology. 2010; 74(Suppl 1): S17-S24, doi: 10.1212/ WNL.0b013e3181c97d99, indexed in Pubmed: 20038758.

49. Juryńczyk M, Craner M, Palace J. Overlapping CNS inflammatory diseases: differentiating features of NMO and MS. J Neurol Neurosurg Psychiatry. 2015; 86(1): 20-25, doi: 10.1136/jnnp-2014-308984, indexed in Pubmed: 25248365. 